\title{
Why black hole production in scattering of cosmic ray neutrinos is generically suppressed
}

\author{
Dejan Stojkovic ${ }^{1}$, Glenn D. Starkman ${ }^{1,2}$, and De-Chang Dai ${ }^{1}$ \\ 1 Department of Physics, Case Western Reserve University, Cleveland, OH $44106-7079$ and \\ 2 Astrophysics Department, University of Oxford, Oxford, OX1 3RH, UK
}

It has been argued that neutrinos originating from ultra-high energy cosmic rays produce black holes deep in the atmosphere in models with TeV-scale quantum gravity. Such black holes would initiate quasi-horizontal showers far above the standard model rate, so that the Auger Observatory would observe hundreds of black hole events. This would provide the first opportunity for experimental study of microscopic black holes. However, any phenomenologically viable model with a low scale of quantum gravity must explain how to preserve protons from rapid decay mediated by virtual black holes. We argue that unless this is accomplished by the gauging of baryon or lepton number, the suppression of proton decay will also suppress quantum gravity mediated lepton-nucleon scattering, and hence black hole production by scattering of ultra-high energy cosmic ray neutrinos in the atmosphere. We discuss explicitly the split fermion solution to the problem of fast proton decay.

PACS numbers: ???

It is widely accepted that black holes can be produced in collisions of particles with center-of-mass (COM) energy $E_{C M}$ larger than the scale $M_{*}$ at which the quantum mechanical nature of gravity is manifest. If two incoming particles collide with $E_{C M}>M_{*}$, and an impact parameter smaller than the gravitational radius corresponding to $E_{C M}$, then a black holes with mass $E_{C M}$ forms. This is of little practical interest in theories where quantum gravity is manifest only at the Planck scale, $M_{P l} \simeq 10^{19} \mathrm{GeV}$. However, the quantum gravity scale could be as low as $1 \mathrm{TeV}[1]$. The exciting possibility is that black holes could then be produced and studied in near-future accelerator experiments [2]. For example, the Large Hadron Collider (LHC), due to start operating in 2007, will have $E_{C M} \simeq 14 \mathrm{TeV}$. Numerical estimates show that it should be able to produce $\mathcal{O}\left(10^{7}\right)$ black holes per year if $M_{\star} \lesssim 1 \mathrm{TeV}$.

In [3, it was pointed out that black holes could also be produced by ultra-high energy cosmic rays interacting with nucleons in the atmosphere, with $E_{C M}>100 \mathrm{TeV}$. These microscopic black holes would decay nearly instantaneously through Hawking radiation, producing extremely energetic cosmic ray showers.

The authors of [3] argued that cosmic neutrinos with energies above $10^{6} \mathrm{GeV}$ are effective sources of black holes, with production cross-sections large enough to be relevant for near-future cosmic ray observatories and well understood fluxes. Neither strong nor electromagnetic interactions degrade the neutrino energy before it interacts quantum-gravitationally, and, since the neutrino interaction length is far longer than the thickness of the Earth's atmosphere, neutrinos produce black holes uniformly at all atmospheric depths. Therefore, the most promising signal for black hole creation by cosmic rays should be quasi-horizontal showers initiated by neutrinos deep in the atmosphere.

It is straightforward to estimate the cross-section for production of a $(4+n)$-dimensional black hole [2]. Consider two particles moving in opposite direction with $E_{C M}=\sqrt{\hat{s}}$. If their impact parameter is smaller than the Schwarzschild radius of a $(4+n)$-dimensional black hole of mass $E_{C M}$,

$$
R_{S} \approx \frac{1}{M_{*}}\left(\frac{M_{\mathrm{BH}}}{M_{*}}\right)^{\frac{1}{1+n}}
$$

then a black hole with mass $M_{\mathrm{BH}}=\sqrt{\hat{s}}$ forms. Thus, the black hole production cross-section is estimated to be the geometric cross-section of the resulting black hole,

$$
\hat{\sigma}(i j \rightarrow \mathrm{BH}) \approx \pi R_{S}^{2},
$$

where $i$ and $j$ denote the two colliding particles. The total black hole production cross-section in neutrinonucleon scattering is thus

$$
\sigma(\nu N \rightarrow \mathrm{BH}) \simeq \sum_{i} \int_{M_{m i n}^{2} / s}^{1} d x \hat{\sigma}_{i}(x s) f_{i}(x, q),
$$

where $s=2 m_{N} E_{\nu} . \quad\left(m_{N}\right.$ is the nucleon mass and $E_{\nu}$ is the neutrino energy.) The sum runs over all partons in the nucleon, with $f_{i}$ the parton distribution functions. $M_{\min }$ is a minimal mass for which this formula is applicable $\left(M_{\min } \sim M_{*}\right)$, while $q$ is momentum transfer. The cross section for black hole production is found to be several orders-of-magnitude higher than the standard model cross-section for $\nu N \rightarrow L X$, if $M_{\min } \approx 1-10 \mathrm{TeV}$.

Quasi-horizontal showers in the atmosphere can be observed by air shower ground arrays or air fluorescence 
detectors. The largest current and near-future cosmic ray experiment is the Auger Observatory. Numerical estimates using (3) indicate that hundreds of such black holes events may be imminently observed at the Auger Observatory in its first five-year run $(2003-2008)$ [3], before LHC data becomes available.

While the production of quantum black holes seems inevitable in particle collisions at center-of-mass energies above the quantum gravity scale, there are important caveats arising from the stability of the proton. The proton lifetime is greater than $2.1 \times 10^{29}$ years [4]. More particularly it is $>10^{33}$ years in the channel $p \rightarrow e^{+} \pi^{0}$, and $>10^{32}$ years in the channel $p \rightarrow \nu \pi^{+}$. (The latter limit is technically from neutron stability inside nuclei; the direct proton limit is an order of magnitude weaker.) In principle, baryon and lepton number violating operators that connect quarks and leptons, of the form QQQL (where Q denotes a quark, while $\mathrm{L}$ denotes either a charged lepton or neutrino), can mediate proton decay. Such operators are expected in both grand unified theories and quantum gravity. Since protons are the lightest baryons, and since leptons are the only free fermions lighter than protons, baryon and lepton number conservation would be violated (assuming electric charge and fermion number are conserved) if a proton could decay.

Baryon and lepton number violating operators do not occur in the perturbative standard model. However, these conservation laws follows from an accidental low energy effective global symmetry, in the sense that all the renormalizable operators that are consistent with the gauge symmetry of the electroweak standard model turn out to conserve baryon and lepton number as well. B and L (thought not B-L) are violated non-perturbatively in the standard model. B and L can also be violated at high energy. For example, quantum gravity is expected to violate all conservation laws that follow from such effective global symmetries [5, [, , 7], nor are they protected by claims of unitarity in black hole evolution [7]. In particular, quantum gravity mediated processes like

$$
p \rightarrow \pi^{0}+e^{+}, \quad p \rightarrow \pi^{+}+\nu \text { and } p \rightarrow \pi^{+}+\bar{\nu},
$$

would be expected to give the proton a life-time $[\underline{5}, \underline{6}$

$$
\tau_{p} \sim m_{p}^{-1}\left(M_{*} / m_{p}\right)^{4} .
$$

( $m_{p}$ is the proton mass.) If $M_{*}=M_{P l}=10^{19} \mathrm{GeV}$ we have $\tau_{p} \sim 10^{45} \mathrm{yr}$, much longer than the current limits. In models with $\mathrm{TeV}$-scale quantum gravity, i.e. $M_{*}=$ $10^{3} \mathrm{GeV}$, one loses 64 orders of magnitude in (5). This results in a disastrously short proton life-time.

Consider the process in which a baryon, $B$, such as a proton, decays to a anti-lepton, $\bar{L}$, such as a positron, or anti-neutrino, plus other particles (which we denote as $\mathrm{X}$ ) that carry no net B or L quantum numbers, via some intermediate quantum gravity state $\mathrm{BH}$ (which we shall call a black hole despite any subtle technical issues),

$$
B \rightarrow \mathrm{BH} \rightarrow \bar{L}+X .
$$

The possibility of (6) implies the possibility of

$$
B+L \rightarrow \mathrm{BH} \rightarrow X .
$$

and vice versa. In (7), the lepton scatters off a baryon and produces a menagerie of particles, $\mathrm{X}$, mediated by the same intermediate quantum gravity state. These same arguments apply to $((B-L)$-violating $)$ proton decays to leptons, implying the possibility of $B \bar{L}$ scattering.

To stabilize the proton, one must somehow forbid (6). There are two approaches. One can prevent the formation of the intermediate black hole, or one can ensure that the black hole decays back to a state with the same baryon or lepton number as the initial state. In order for the black hole decay to depend on the properties of the pre-existing initial state, either the black hole or its environment would itself need to carry the information about the quantum numbers of the initial state.

For the black hole itself to remember the initial preblack-hole initial state, it must carry B or L. This requires that we promote the $\mathrm{U}(1)$ invariance associated with B or L from a global to a local symmetry - i.e. that we gauge $U(1)_{B}$ or $U(1)_{L}$ invariance. If $\mathrm{B}$ is gauged then, although the the B-violating processes (6) and (7) are prohibited, the $\mathrm{B}$ conserving processes

$$
\begin{gathered}
B+L \rightarrow \mathrm{BH} \rightarrow B+X, \quad \text { and } \\
B+L \rightarrow \mathrm{BH} \rightarrow B+L+X
\end{gathered}
$$

are permitted. Similarly, if L is gauged then, although (6) and (7) are prohibited, the L conserving processes

$$
B+L \rightarrow \mathrm{BH} \rightarrow L+X
$$

and (9) are permitted. Finally, even if both B and L are gauged, the B and L conserving process (9) is allowed.

Gauging B or L permits cosmic ray neutrinos scattering off atmospheric nucleons to produce black holes and yet protects the proton from decay. However, gauging $\mathrm{B}$ or $\mathrm{L}$ has proven to be problematic. If $U(1)_{B}$ were an unbroken gauge symmetry, there would be a long range interaction not seen in experiments. Therefore, $U(1)_{B}$ needs to be broken down to a discrete gauge symmetry. The leftover discrete symmetry can preserve baryon number modulo some integer [9]. To suppress dangerous $n \rightarrow \bar{n}$ oscillations [8] one must forbid both $\Delta B=1$ and 2 operators. The lowest allowed operators would then be $\Delta B=3$ (which are of dimension 12 and higher). The most common problem is arranging for cancellation of gauge anomalies 10]. This requires either an unusual charge assignment to existing particles or the existence of new exotic particles. There are other problems related 
to the idea of gauge couplings unification. Although one cannot exclude gauging $U(1)_{B}$ or $U(1)_{L}$, one must allow for the possibility that they are not, and that the stability of the proton in low-scale quantum gravity models is due to other causes.

The second possibility is for the black hole's environment to preserve the information about the pre black hole initial state. The only thing that distinguishes the place where the black hole was created from any other is that it is on the brane. Thus for the environment to inhibit B or $\mathrm{L}$ violating black hole decay, there must be different locations in the bulk for baryons or leptons. This is precisely the split fermion model [11]. We shall find that in this model, black hole production is heavily suppressed.

As an alternative to gauged baryon number, 11 proposed that standard model fields are confined to a "thick" brane - much thicker than $M_{*}^{-1}$. Quarks and leptons are stuck on different three-dimensional slices within the thick brane (or on different branes), separated by much more than $M_{*}^{-1}$. This separation causes an exponential suppression of all direct quantum-gravity couplings between quarks and leptons, due to exponentially small wave functions overlaps. The virtual black holes associated with protons therefore have an exponentially small probability of decaying with emission of leptons [12], and thus of mediating lepton number violation. Assuming that black holes do not mediate electric charge nonconservation or fermion-number violation, this stabilizes the proton. For appropriately chosen spatial separation $d$ between the quarks and leptons, the proton decay rate can, as we shall see, be safely suppressed.

The propagator between fermions which are separated in the extra dimension was derived in [11]:

$$
P_{d}(t) \approx(\sqrt{2 \pi} R /(\sigma t)) \exp \left[-d^{2} /\left(2 \sigma^{2}\right)\right],
$$

$R$ is the characteristic size of the extra dimension $(d \sim R)$ and $\sigma$ is the inverse width of the fermion wave function in the extra dimension. The momentum transfer of $t$-channel scattering is $\sqrt{-t}$. The expression in (11) is valid in the limit of large momentum transfer, $\sqrt{-t} \gg \max \left(R^{-1}, d / \sigma^{2}\right)$. The propagator has the usual four-dimensional momentum dependence save that the coupling is suppressed by the exponentially small wave function overlap. The proton decay width is proportional to the propagator squared. To adequately suppress proton decay, we need to increase the lifetime by $\mathcal{O}\left(10^{51}\right)$. This implies that $e^{-d^{2} /\left(2 \sigma^{2}\right)} \sim 10^{-25.5}$, or a rather modest hierarchy $d \sim 10 \sigma$. It is obvious that black hole production via such direct tree level quark-lepton interactions will be heavily suppressed.

What about higher order effects? Gauge bosons mediate lepton-quark interactions, so might enable proton decay and black hole production. The inability of gauge bosons to carry fermion number between branes prevents their contributing to proton decay, but not to black hole production.

Gluons might be localized on the same brane as quarks in which case there will be the same exponential suppression of interactions with the leptons, as in the tree level lepton-quark interactions. Alternatively, the gluons may propagate freely between the quark and lepton branes. Standard model interaction between leptons and gluons are of course suppressed because leptons carry no color. However, leptons could, in principle, interact directly with gluons via quantum gravitational interactions. Similarly leptons could interact directly with the weak gauge boson $\left(W^{ \pm}\right.$or $Z$ ) or photon content of a nucleon to make a black hole. (Or the gauge boson content of the leptons could interact with the quarks of the nucleon, or finally the gauge boson content of the leptons could interact with that of the proton.) This too, however, is suppressed compared to expectations.

There are two separate limits to consider - hard gauge bosons and soft gauge bosons. Although the hard gauge boson content of the nucleon near the quark brane is considerable, this falls rapidly as one moves deep into the the thick brane. There is first of all a volume suppression to the cross section, proportional to the ratio of the lepton brane volume to the volume of the full bulk, $(\sigma / d)^{n}$, where $n$ is the number of extra dimensions (for realistic models one usually takes $n \geq 2$, however recent experimental limits seem to force $n \geq 3$ ). There is a further exponential suppression since the harder the virtual gauge bosons, the closer to on-shell it must be. Only ones very nearly on-shell can propogate the large distance between branes. However, the gauge bosons are getting more massive as they move into the bulk (due to the effect of their Kaluza-Klein towers) and the propagator from the plane where they live (with their bare masses) into the bulk is highly suppressed [1].

Soft gauge bosons more readily negotiate the interbrane gulf, after all they mediate standard model interactions, but they are insufficiently energetic to contribute much to the center of mass energy. In order to make a black hole in a scattering they must encounter a much higher energy neutrino. The neutrino flux is a rapidly falling function of energy, so the rate of black hole production by this mechanism is further suppressed (in addition to the above mentioned volume suppression factor).

The interaction between leptons and the Kaluza-Klein graviton content of a proton faces the same issue as between leptons and the protons's gauge boson content. And will be at least suppressed by the same geometric factor that applies for delocalized gauge bosons, i.e. $(\sigma / d)^{n} \simeq 10^{-n}$.

So, the production of the small black holes whose radius is much smaller than the quark-lepton separation will be suppressed. What about larger black holes that can bridge the gap between the fermions? Their production will be also suppressed. To see this, note that the Schwarzschild radius of a higher dimensional black hole 
grows very slowly with its mass, $R_{s} \propto M_{B H}^{1 / 1+n}$. In order to overcome the large $\left(\sim 10 M_{*}^{-1}\right)$ quark-lepton separation, one requires COM energies larger by a factor of $10^{n+1}$ than otherwise expected, and therefore incident neutrino energies larger by $10^{2(n+1)}$. The neutrino spectrum at these high energies is believed to be falling as $\sim 1 / E^{3}$ which gives a suppression of $\sim 10^{18}$ for two extra dimensions, and much more for more extra dimensions.

Thus, all the potential black-hole producing interactions are suppressed by wave function overlap factors, whether exponential or geometric. Black hole formation will thus be well suppressed in the split-brane model.

As an illustration, in Fig. 11 we plot the black hole production cross sections for the two cases (non-split and split fermions). For the split fermion case, we use the equation (3), but instead of (2) we use $\hat{\sigma} \approx \pi\left(R_{s}^{2}-d^{2}\right)$, where $d$ is the separation between the fermions. Gauge bosons are allowed to propagate between the fermions. This takes into account only a simple wave function overlap suppression (no eventual loop suppression, energy lost to gravitational radiation etc.). Even in the most optimistic case of two extra dimensions the suppression factor is of the order of $10^{3}$, in good agreement with the previous discussion. The suppression will grow stronger with more extra dimensions and with larger separation between the fermions.

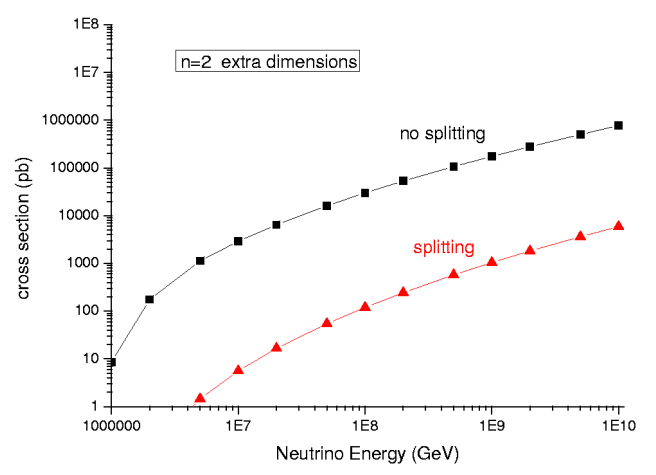

FIG. 1: The black hole production cross section for the conventional (non-split) and split fermion models. We take $n=2$ extra dimensions, the fermion wave function width $\sigma \sim M_{*}^{-1}$ and the separation between the quarks and leptons $d \sim 10 M_{*}^{-1}$, where $M_{*}=1 \mathrm{TeV}$.

We note that the splitting of leptons from quarks would not suppress $\Delta B=2$ processes, like $n-\bar{n}$ oscillations [8], large left-handed Majorana masses for fermions, and large mixing between the neutrino generations. That would require, for example, further splitting between uptype and down-type quarks, or splitting between the different lepton generations. However, the purpose of this paper is not to fix the split-fermion model, but to use it as a simple explicit illustration of a model where black hole production in lepton-nucleon scattering is suppressed.
We see that the correct black hole production cross section in collisions of neutrinos and nucleons is not given by (3). Large suppression factors, ranging from wave function overlaps of $10^{51}$ for direct tree-level processes, to more modest but still substantial volume overlap factors $10^{n}$ (with $n \geq 2$, or rather $n \geq 3$ in the light of the latest experimental limits) for gauge boson-lepton processes, divide the geometrical cross section (2) and thus enter the total production cross section (3) as well. This renders the corresponding probability for black hole production by cosmic neutrinos uninteresting for the Auger Observatory.

The final possibility is that production of the intermediate black hole in (6) is forbidden. In this case, not only is (6) suppressed, but so are all black-hole mediated scattering processes between a proton and a lepton or anti-lepton. We therefore learn that, in the context of low-scale quantum gravity, unless the stability of the proton is due to the gauging of $\mathrm{B}$ or $\mathrm{L}$, neutrino-nucleon scattering (and antineutrino-nucleon scattering) does not result in copious production of black holes.

We note that black holes might still be produced in atmospheric nucleon-nucleon or photon-nucleon scattering. In the split fermion model, nucleon-nucleon scattering arises because quark wave functions are not separated from themselves. (Even if every flavor of quark was on a different brane, the same quark flavor from both nucleons can scatter.) Photon-nucleon scattering arises because the photons are not confined to one or another brane. However, these process will lead to completely different production cross sections and are much less certain. (The flux of cosmic neutrinos used in [3] comes from the decay of $\pi^{ \pm}$in collisions of ultra-high energy protons with the cosmic microwave background.)

Furthermore, the Earth's atmosphere is not transparent to nucleons or photons as it is to neutrinos. The typical nucleon or photon will scatter by ordinary Standard Model processes many times before it has a chance to scatter quantum gravitationally. In so doing, it will typically lose too much energy to produce a black hole. Only the rare photon or nucleon will scatter quantum gravitationally early enough in the shower process to produce a black hole. Moreover, even if the cosmic ray nucleon or photon flux, and the scattering cross section are large enough to produce detectable numbers of black holes, the experimental signature would be much different than for those created in neutrino-nucleon scattering. In particular, one can not expect quasi-horizontal showers deep in the atmosphere. Instead, these black holes would be produced high in the atmosphere. The experimental signatures and expected rates merit further careful review, but the prospects for detection are considerably less optimistic than had been calculated for neutrinos.

The other possibility is to have black holes produced in lepton-lepton (for example $\nu e^{-}$) scattering in the atmosphere. From eq. (3) we see that the total black hole 
production cross section depends on the COM energy squared in the collision, which in turn is proportional to the mass of the target. Since the electron mass is 2000 times smaller than the proton mass, the threshold neutrino energy for leptonic black-hole production should be 2000 times higher. This implies a significant reduction in flux of neutrinos above threshold, since the flux goes down steeply with energy $\left(\sim 1 / E^{3}\right)$. Since using the naive calculation of neutrino-nucleon scattering one expected to detect a hundred black hole events in a five-year run of the Auger Observatory, therefore the expected suppression of $\approx 10^{9}$ in the neutrino-electron case renders the rate of black hole production completely uninteresting in terrestrial detectors.

In conclusion, we reconsidered the question of black holes production by cosmic neutrinos in models with $\mathrm{TeV}$-scale quantum gravity, originally considered in 3 ]. We pointed out that, unless baryon number or lepton number arise from gauge symmetries, the stability of the proton suggests that lepton-nucleon scattering does not lead to black hole production that might be observable at the Auger. As an example, we showed that in the model of split fermions, the rate of black hole production by cosmic neutrinos is suppressed to an uninteresting level. Although the possibility of black hole production by high energy cosmic ray nucleons or photons still remains, the fluxes are uncertain, and the signatures and rates need to be properly reconsidered but are pessimistic.

Acknowledgements: The authors are grateful to Tanmay Vachaspati, Francesc Ferrar, Jonathan Feng, Alfred Shapere, Manuel Toharia and Luis Anchordoqui for very useful conversations. The work was supported by the DOE grants to the Michigan Center for Theoretical Physics, University of Michigan, and to the particle as- trophysics group at Case Western Reserve University.

[1] N. Arkani-Hamed, S. Dimopoulos and G. Dvali, Phys. Lett. B429, 263 (1998); L. Randall and R. Sundrum, Phys. Rev. Lett. 83, 3370 (1999);

[2] S. B. Giddings and S. Thomas, Phys. Rev. D65 056010 (2002); T. Banks, W. Fischler, hep-th/9906038 ; S. Dimopoulos, G. Landsberg, Phys. Rev. Lett. 87161602 (2001); D. Stojkovic, Phys. Rev. Lett. 94011603 (2005); JHEP 0409061 (2004); V. Frolov, D. Stojkovic, Phys. Rev. Lett. 89151302 (2002); Phys. Rev. D66 084002 (2002);Phys. Rev. D67 084004 (2003); Phys. Rev. D68 064011 (2003)

[3] J. L. Feng, A. D. Shapere, Phys. Rev. Lett. 88021303 (2002)

[4] S. Eidelman et al., Review of Particle Physics, Phys. Lett. B592 (2004).

[5] S. Hawking, Phys. Rev. D53, 3099 (1996)

[6] F.C. Adams, G.L. Kane, M. Mbonye and M.J. Perry, Int. J. Mod. Phys. A16, 2399 (2001)

[7] D. Stojkovic, G.D. Starkman, F.C. Adams, Gravity Essay Competition 2005, to apper in the special Essay issue of Int. J. Mod. Phys.

[8] C.E. Carlson and C.D. Carone, Phys. Lett. B512, 121 (2001); K. Benakli and S. Davidson, Phys. Rev. D60, 025004 (1999).

[9] L.M. Krauss and F. Wilczek, Phys. Rev. Lett. 62, 1221 (1989).

[10] L.E. Ibanez and G.G. Ross, Nucl. Phys. B368, 3 (1992).

[11] N. Arkani-Hamed, M. Schmaltz, Phys. Rev. D61 033005 (2000); N. Arkani-Hamed, Y. Grossman, M. Schmaltz, Phys. Rev. D61 115004 (2000)

[12] T. Han, G. D. Kribs and B. McElrath, Phys. Rev. Lett. 90, 031601 (2003). 